\title{
Le récit de voyage au Maroc d'Edmond Picard : au carrefour des genres et au service de la haine
}

\section{Maria Chiara Gnocchi}

\section{(2) OpenEdition}

\section{Journals}

\section{Édition électronique}

URL : http://journals.openedition.org/rief/1477

DOI : 10.4000/rief.1477

ISSN : 2240-7456

\section{Éditeur}

Seminario di filologia francese

\section{Référence électronique}

Maria Chiara Gnocchi, « Le récit de voyage au Maroc d'Edmond Picard : au carrefour des genres et au service de la haine », Revue italienne d'études françaises [En ligne], 7 | 2017, mis en ligne le 15 novembre 2017, consulté le 19 avril 2019. URL : http://journals.openedition.org/rief/1477 ; DOI : 10.4000/ rief. 1477

Ce document a été généré automatiquement le 19 avril 2019.

\section{(†) $\odot$}

Les contenus de la RIEF sont mis à disposition selon les termes de la Licence Creative Commons Attribution - Pas d'Utilisation Commerciale - Pas de Modification 4.0 International. 


\title{
Le récit de voyage au Maroc d'Edmond Picard : au carrefour des genres et au service de la haine
}

\author{
Maria Chiara Gnocchi
}

1 Tous ceux qui se sont penchés sur la vie littéraire et artistique belge de la fin du XIX siècle ont nécessairement rencontré le nom d'Edmond Picard (1836-1924). Avocat, professeur de droit, à un moment sénateur socialiste, mais aussi écrivain, journaliste, amateur d'art, mécène, animateur de la vie culturelle bruxelloise, il est l'auteur de nombreux articles, de quelques essais, de plusieurs pièces de théâtre, de deux récits de voyage ; il est célèbre pour avoir fondé la revue L'Art moderne et théorisé l'« âme belge »1. Ce portrait aux multiples facettes est pourtant incomplet: Edmond Picard a également été un théoricien débridé de l'antisémitisme et du racisme. Ce fait, longtemps occulté ou minimisé ${ }^{2}$ a attiré l'attention, dans les vingt dernières années, de différentes personnalités (Michel Graindorge, Foulek Ringelheim, Paul Aron et Cécile Vanderpelen-

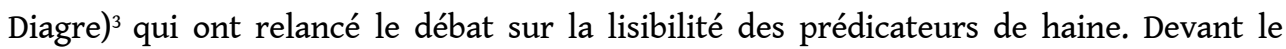
caractère intenable des discours de Picard, le critique doit choisir : soit il détourne les yeux, soit il se penche sur les textes qui ont véhiculé la haine pour comprendre leur fonctionnement et leur pouvoir de séduction. C'est cette dernière attitude que j'ai choisi d'adopter, me concentrant sur El Moghreb al Aksa, un récit de voyage au Maroc que Picard publie en $1889^{4}$, dans lequel l'auteur combine racisme et antisémitisme d'une façon assez originale et qui se révèlera une matrice importante pour bien d'autres œuvres de sa plume, voire un modèle pour d'autres auteurs.

2 El Moghreb al Aksa, dont des extraits paraissent déjà en 1888 dans La Société nouvelle ${ }^{5}$, est le premier texte dans lequel Picard développe sa théorie des races, et il peut en même temps être considéré comme le volet inaugural d'une sorte de dossier antisémite que complètent les essais Synthèse de l'antisémitisme (1892), Contribution à la révision des origines du christianisme (1892), L'Aryano-sémitisme (1898), ainsi que la pièce Jéricho : comédie-drame en trois actes et XXIV scènes (1902). On peut ajouter à ces œuvres un syllabus du cours de droit que Picard donne à l'Université nouvelle à partir de 1890, Le Droit pur, qu'il publiera dans 
différentes versions jusqu'en 1910. L'auteur développera ultérieurement sa théorie des races dans En Congolie, son récit de voyage au Congo ${ }^{6}$.

Picard n'a sûrement pas inventé la théorie des races ; ni l'antisémitisme ni la perception des Africains comme des êtres inférieurs n'étaient source de grand scandale à l'époque. On peut par contre affirmer qu'un écrivain de son envergure a certainement contribué à diffuser ces idées auprès d'un large public. En effet, ses textes sont lus et influencent largement d'autres écrivains, ainsi que le public des lecteurs. Si les récits de voyage au Congo étaient déjà assez nombreux en Belgique, à l'époque où Picard publie En Congolie, on peut inversement affirmer qu'El Moghreb al Aksa a eu un rôle fondateur dans la relation de la littérature belge à l'espace nord-africain ${ }^{7}$. Il est vrai que le récit parait d'abord en tirage réduit et que les précieuses illustrations qui l'accompagnent en font un livre confidentiel, mais le livre sera réédité quatre ans plus tard, dans une version courante sans illustrations, abordable par un public beaucoup plus vaste. Il ne faut pas sousestimer l'influence que pouvait avoir, à l'époque, le fondateur de la célèbre revue L'Art moderne. Le rayonnement de la parole de Picard dépasse le domaine de la littérature : son récit a largement inspiré la peinture de Théo Van Rysselberghe, qui voyage avec lui et qui illustre la première édition de son livre ${ }^{8}$, mais aussi celle de Félicien Rops, qui emporte avec lui El Moghreb al Aksa lors de son voyage en Afrique du Nord en 1889'. Van Rysselberghe - qui n'est pas encore, à l'époque, le grand-père de la fille d'André Gide influence à son tour Émile Verhaeren, avec qui il entretient une intense correspondance. Pour finir, on peut se demander si Pierre Loti a lu le texte de Picard, lui qui publie en 1890 - une année après El Moghreb al Aksa - une œuvre intitulée Au Maroc qui est, comme celui de Picard, à la fois un récit de voyage personnel et un récit d'ambassade ${ }^{10}$.

Cette étude ne veut être ni une condamnation, ni une tentative de justification. Aucun jugement de valeur dans ces lignes, mais une analyse à la fois rhétorique et thématique. Les questions auxquelles je vais essayer de répondre sont les suivantes : quelle est la clé du succès d'El Moghreb al Aksa ? De quelle manière le discours raciste et antisémite y est-il décliné ? Peut-on parler de haine à propos de ce récit? En cas de réponse affirmative, quelle forme prend-elle, quelles images incarne-t-elle ? Et pourquoi, malgré tout, a-t-on longtemps pu parler de Picard en délaissant cet aspect de son œuvre?

\section{El Moghreb al Aksa : beaucoup plus qu'un coup de tête}

5 Un mot, pour commencer, sur les circonstances du voyage que Picard entreprend entre décembre 1887 et mars 1888, et qui est à l'origine de son œuvre, dont je disais que c'est aussi un récit d'ambassade. À l'époque, le roi des Belges, Léopold II, vient d'acquérir l'État indépendant du Congo et cherche une sorte de pied-à-terre en Afrique pour faciliter ses échanges avec le Congo ; c'est pourquoi il invite le baron Whettnall, ministre résidant de Belgique à Tanger, à prendre des accords avec le sultan Moulay Hassan, qui régnait alors sur le Maroc. Edmond Picard, qui voyage à ses frais, fait partie de la délégation qui accompagne le baron Whettnall.

6 À peine parti de Bruxelles, l'auteur entame un journal qui s'étoffe jour après jour et qui composera bientôt un riche volume de plus de 400 pages. Lorsqu'il le publie, il le fait débuter par quelques phrases invitant à le considérer comme une œuvre tout à fait personnelle, née à la suite d'un coup de tête. Picard explique qu'il s'est décidé à partir enlevé, "par coup de passion », par la fantaisie (p.9), et qu'il a écrit la relation de ce 
voyage pour lui-même, ou tout au plus pour ses amis, oubliant ces «facteurs amoindrissants » que sont « la Rhétorique, le Public, la Critique » (p. 7) ${ }^{11}$. Il est vrai que le texte paraît d'abord en tirage confidentiel, mais primo, le livre sera bientôt réédité dans une version courante, et secundo, une étude fouillée du texte permet d'affirmer que $\mathrm{El}$ Moghreb al Aksa est beaucoup plus que le résultat d'un coup de tête. La dénégation du souci à la fois esthétique et idéologique a l'air d'une prétérition: Picard prête en réalité beaucoup de soin à la composition de son œuvre, ce que prouvent les nombreux commentaires métanarratifs ponctuant le texte. Et d'ailleurs, si j'ai décidé de prendre en examen ce texte, c'est précisément parce que la rhétorique n'y a pas vraiment été oubliée, et qu'elle y joue au contraire un rôle important, au profit de la haine.

$7 \quad$ Il me semble qu'on peut repérer dans le texte de Picard une série de représentations, plus ou moins imagées, au service de la haine, accompagnées et même agencées par des stratégies discursives finalisées à la dénégation de cette même haine - ce qui est, bien entendu, une manière très efficace de la soutenir. Parmi les premières, je distinguerai les représentations complexes, générales, de l'altérité sémite, et les images (plus ou moins stéréotypées, toutes généralement dépréciatives) que l'auteur donne des sujets arabes et juifs.

\section{Représentations de l'altérité sémite}

8 Passé Gibraltar, arrivé à Tanger, Picard a l'impression de débarquer sur « une autre planète ». Tout ce qu'il voit lui apparait sous le signe de l'altérité, et cette première impression ne change pas au cours des trois mois qu'il passe au Maroc: une vingtaine de jours avant d'en repartir, cette terre reste pour lui « le pays des Mystères» (p. 324). Ce n'est pas pour rien qu'il la désigne, dans le titre de son récit, El Moghreb al Aksa, c'est-àdire le Maghreb (ou le Maroc) extrême ou, puisque Maghreb signifie Occident en arabe, «le Far West», et donc une terre lointaine, quelque peu mythique et fantasmée. La civilisation marocaine lui apparaît comme l'envers de celle à laquelle il sent appartenir, et ce qui rend tout dialogue impossible est l'écart dû aux différences raciales :

Quelle leçon d'histoire, d'ethnologie, de philosophie que ce voyage [...]! [...] Une autre race : j'avais entendu prononcer ces mots et croyais les comprendre; mais je n'avais point senti, comme par le présent contact, la réalité de l'abîme auquel ils correspondent, et l'impossibilité de le combler. Ah! le rêve de ceux qui croient à l'unité humaine, à la possibilité des transformations, à la conversion d'un Sémite en Aryen! (p. 124-125)

On conçoit, dès lors, l'importance de ce voyage pour l'élaboration des théories de l'auteur. En premier lieu, au Maroc Picard fait l'expérience directe, presque sensorielle d'une incompatibilité entre les races dont il avait jusque-là entendu parler de manière abstraite et qu'il présentera désormais, inversement, comme une donnée objective, comme quelque chose qu'il a vu et senti. En deuxième lieu, il apprend à distinguer la civilisation de la race. Lorsqu'il constate l'abîme qui le sépare de la population marocaine, Picard ne fait pas spécialement allusion à l'islam ou à la culture arabe : la race en serait la véritable responsable. Or, au Maroc Picard ne voit pas que des arabes : il voit des juifs aussi, qui appartiennent à la race sémite comme les arabes et qu'il décrit souvent (on le verra) de manière similaire : les "Beni-Israël », affirme-t-il, sont " aussi barbares que les Maures, Maures eux-mêmes par le sang et par la nationalité » (p. 384). 
10 Les arabes et les juifs finissent par se confondre, alors que la race aryenne et la race sémite font preuve d'un «irrémédiable désaccord » aux yeux de notre auteur (p. 269). La psychologie des sémites est "indéchiffrable» pour les aryens, leurs visages sont « impassibles », leurs âmes " impénétrables » (p. 325). Si la conversation est difficile entre le Pacha et le ministre belge, c'est «moins à cause de la langue que de l'abîme des idées et des sensations béant entre les races»(p.160). On aura remarqué tous ces adjectifs débutant par le préfixe "in", désignant une situation que rien ne peut changer: « irrémédiable », « indéchiffrable », « impassible », « impénétrable »... Picard en arrive à affirmer que le récit de ce voyage lui pose problème en termes de «traduction » : il doit « trouver les mots véhiculaires des choses sémites en des cerveaux aryens » (p. 76).

11 Il faut préciser que, à partir de la deuxième moitié du XIX siècle, des formes d'« antisémitisme racial » s'affirment en France, mais aussi en Belgique et en Allemagne, qui remplacent l'antisémitisme ethnique plus classique ; c'est un antisémitisme fondé sur une sorte de "racisme scientifique " qui doit beaucoup aux théories évolutionnistes dominant le discours scientifique contemporai $\mathrm{n}^{12}$. Picard, qui avait toujours perçu les juifs comme " autres », comme des intrus en Europe, comme les responsables d'une crise sociale qu'il réprouvait, lui qui l'année précédente, en 1886, avait lu avec beaucoup d'intérêt La France juive de Drumont (mais qui n'avait jamais fait preuve d'antisémitisme dans ses œuvres), il trouve au Maroc la raison de son intolérance vis-à-vis des juifs côtoyés en Europe: c'est qu'ils ne sont pas à leur place, alors que l'Afrique du Nord enfin, tout ce pan de terre qui part du Maroc et arrive jusqu'à la péninsule arabe - est la terre où tout semble leur convenir. Irréductible, l'altérité sémite est en effet tout aussi inassimilable. Lorsque Picard remarque que la véritable place pour les sémites, tous les sémites, est sur ces terres, il suggère en même temps qu'elle n'est pas en Europe, où l'accord avec la race aryenne est impossible à trouver ; et cela vaut pour les juifs aussi, et même surtout pour les juifs, du moment qu'ils ont tendance à «vivre de la substance d'autrui ", comme il ne manquera pas de souligner (cf. infra). La seule solution qui se profile est l'exclusion ${ }^{13}$ - et c'est ce que Picard développera plus tard par exemple dans Jéricho, où il présente le sionisme comme la seule issue sensée pour les juifs européens.

\section{Figures de la haine : les images dépréciatives}

Voyageur privilégié, Picard se doit de montrer à ses lecteurs ces "autres " qu'il rencontre. Pour ce faire, il recourt à une série d'images dont une partie n'a rien de bien nouveau, mais qui sont agencées dans leur ensemble de manière à servir son discours au profit de la haine.

13 Parmi les images les plus courantes, et les plus stéréotypées aussi, on trouve dans $E l$ Moghreb al Aksa celles qui assimilent les sémites (arabes et juifs) aux animaux, et qui vont souvent de pair avec l'allusion à la malpropreté. Les arabes sont décrits dans leurs vêtements amples qui « dissimul[ent] l'imperfection de leurs corps secs, sans harmonie, maigrement musclés, ayant les tendons de l'animalité et sa vigueur, mais non les contours arrondis d'une humanité plus haute » (p. 123). Le kaïd de la Kasbah de Meknès est décrit comme un «monstrueux personnage »:

[...] court, le visage énorme, yeux, nez, lèvres, oreilles démesurés, face d'idole grossière taillée à coups de hache dans une souche de têtard par des anthropophages, mains velues et formidables d'orang-outang, suscitant le songe d'accouplements étrangers entre les troglodytes et les animaux primitifs. (p. 278) 

du plaisir à s'épouiller réciproquement comme des singes (p. 70). Dans les deux cas, orang-outang ou singe, le sémite est décrit comme un sous-homme, comme l'animal le plus proche de l'homme, mais à un niveau inférieur dans la chaine évolutive. Simiesque serait aussi la volonté des juifs d'imiter l'Européen, ce que Picard stigmatise lorsqu'il observe «des juifs, dont un vêtu à l'européenne, le chapeau de l'odieuse forme melon » (p. 94). L'auteur tente de nuancer une haine qui ne s'affirme pas moins : par un double transfert qui tient de l'hypallage et de la métonymie à la fois, l'objet qui suscite le rejet ne serait pas le juif, même pas son chapeau, mais la "forme melon" de ce dernier. Et encore : il ne dit pas son agacement vis-à-vis du chapeau : c'est sa forme qui est haïssable. Picard a beau dire qu'il a oublié ce «facteur amoindrissant » qu'est la rhétorique, on voit bien que son expérience à la fois des livres et des tribunaux rend cette séparation difficile.

On peut repérer une allusion (indirecte) aux singes, ou en tout cas à des sous-hommes qui imitent la race supérieure, dans la description que Picard fait de l'école juive du Mellah de Fès. L'appréciation du travail du directeur de l'école se fait dans ces termes: «Certes Salomon Benoliel réussit le dégrossissement de ces barbares. Il les fait bien marcher sur leurs pattes de derrière. - Ils ne savaient pas même s'asseoir quand j'ai commencé, dit-il. - » (p. 385). encore une fois très courantes, par exemple celle du juif "sangsue ", qui «suce et se gorge » (p. 245), «vivant de la substance d'autrui » (p. 95). Ou alors, Picard connote de manière négative la couleur noire, souvent choisie par les juifs pour s'habiller : «noir, couleur de malédiction, couleur de malheur » (p. 109) ; faisant allusion à leurs calottes également noires, l'auteur ajoute, plus loin : «la couleur qui n'existe pas, à laquelle la nature répugne » (p. 386).

Évidemment, le fait qu'il s'agisse de stéréotypes ne réduit pas l'intérêt de leur analyse ; les lieux communs ne naissent pas en tant que tels : communs, ils le deviennent, et cela grâce aux discours qui les réitèrent leur attribuant un sens qui, de discours en discours, se fige et s'affirme ${ }^{14}$. De même, il serait inutile, voire nuisible, de minimiser la haine de Picard en la reconduisant à ce qu'on appelle l'« air du temps ». S'il est important de situer toute attitude dans son contexte ${ }^{15}$, il est tout aussi vrai que l'air du temps est souvent un effet de perspective : il ne préexiste pas aux textes, ou en tout cas les textes contribuent à le façonner, et c'est pourquoi il est important de les analyser dans leur fonctionnement, dans leur pouvoir créateur.

Les images sont mises au service d'un discours qui s'appuie, on l'a vu, sur l'opposition entre aryens et sémites, ce dernier ensemble comprenant à la fois les juifs et les arabes. On remarque dès lors dans le texte de nombreuses images présentant de manière similaire les juifs et les arabes. Par exemple, lorsqu'il lit la description d'un kaïd arabe, comparé à un renard, qui « se révèle en sa foncière duplicité, menteur comme tous ici, caressant en apparence, mauvais au fond, rusé, cupide » (p. 415), le lecteur a toutes les chances de l'associer aux juifs, décrits dans le même récit comme des "corsaires", « jouant les bons apôtres [...], soutireurs, accapareurs ", « caressant pour obtenir, habiles pour conclure, insolents pour exiger, impitoyables pour exécuter » (p. 130-131).

Dans la description du Kaïd Messmoudi, qui guide la délégation belge à partir d'un certain moment, on peut reconnaitre un amalgame de traits attribués traditionnellement aux arabes et aux juifs (ce n'est pas pour rien que Picard reconnaît en lui un « superbe type de 
sémite »). D'une part, il est décrit comme "[1]e terrible Messmoudi, à la tête de négrier, au torse de boucher", comme un "démon" possédé d'une "fureur satanique " (p. 189-190) : ce sont les clichés qui identifient l'arabe aux yeux de l'Européen. On sait bien, grâce entre autres aux travaux sur l'« orientalisme » d'Edward Saïd, à quel point la démonisation de l'arabe et de l'islam font partie des stéréotypes qui ont filtré le regard européen porté sur le monde arabe ${ }^{16}$, et le discours colonial ne fait que renforcer ces images, puisque les Européens ont tendance à présenter leur intervention en Afrique noire comme un acte humanitaire voué à sauver les indigènes des esclavagistes arabes (il $\mathrm{y}$ a toute une littérature là-dessus $)^{17}$. D'autre part, Messmoudi est présenté «pieds et jambes nus, les orteils agrippant le sol avec des morsures brusques de griffes ", ce qui, audelà de la métaphore animale, fait plutôt penser à un autre stéréotype, celui des juifs aux « doigts crochus " (et avides), image ambiguë que renforce l'allusion au visage «mangé par une barbe noire de Samson » : si le visage maigre et barbu appartient à l'imaginaire partagé relatif tant aux arabes qu'aux juifs, la référence à Samson, héros de l'Ancien Testament, fait bousculer le portrait du côté des juifs. Et d'ailleurs, cette image sert bien une autre théorie de Picard, selon laquelle ce ne serait pas le Nouveau Testament, mais le Coran à compléter l'Ancien Testament. Encore une fois, une image, fruit de l'observation directe de l'auteur, est mise au profit d'une théorie qui sera développée seulement plus tard.

Le texte présente d'autres images sans doute moins agressives, du moins en surface, qui traduisent toutefois un mépris haineux encore plus marqué. Par exemple, Picard évoque souvent le caractère simple, primitif, rudimentaire de la civilisation marocaine, qui serait "en équation avec les forces intimes, bornées, du Sémitisme» (p. 119). Pourquoi bornées? Parce que le droit, les mœurs, toutes les attitudes des Marocains seraient influencées par les dimensions du crâne sémitique, qui est plus petit que le crâne aryen et contient donc un cerveau plus exigu, voire « inconsistant» (p. 181). De même, «la fixité de leur religion rudimentaire " serait "bien faite pour leur race à cervelle angustiée " (p.58). Picard invite à se méfier de ces gens, parce que «[c]omme toutes les cervelles étroites, la cervelle sémite a peu de prévision et peu de souvenir » (p. 122-123). Il résume ainsi les traits de la race :

Race stagnante, hostile aux changements, défiante, heureuse en son sémitisme étroit et sobre, préférant d'instinct sa vie satisfaite d'une simplicité de troupeau où quand il y a souffrance les corps seuls pâtissent, à la nôtre où ce sont les âmes insatiables, où les innombrables minuscules besoins de l'existence bourgeoise tuent les deux ou trois grands instincts de ces primitifs si près du fauve. (p. 288)

21 Picard dit avoir laissé de côté la rhétorique, qui semble toutefois bien opérer dans ce texte dans lequel différents éléments se répondent. On aura remarqué, dans cette dernière citation, qu'un lien est établi entre l'«étroitesse", à la fois anthropométrique et intellectuelle, des sémites, leur méfiance à l'égard des changements mais aussi leur animalité, leur préférence pour la «simplicité de troupeau » et leurs instincts bestiaux. C'est une idée sur laquelle l'auteur reviendra à plusieurs reprises dans le texte :

Pourquoi l'immuabilité, pourquoi le fatalisme? La race ! La race intelligente mais à étroites parois. La race à vision claire mais sans portée. La race à humanité sans lointains, essentiellement rudimentaire dans ses conceptions sociales, sans le progressif indéfini, sans l'aptitude aux analytiques spéculations intellectuelles du haut aryanisme inépuisablement éducable [...]. (p. 396)

Pour finir, Picard opère une dépréciation systématique de toute la culture locale : à ses yeux, il n'y aurait en fait pas de culture au Maroc: pas de livres, pas de journaux (p. 325), 
une musique indigne de ce nom (p. 331-332), etc. L'auteur en arrive à déclarer que l'art n'aurait porté de fruits que lorsque «l'élément aryen n'avait pas encore disparu des terres gagnées à l'Islam» (p. 392) ; «l'Arabe n'a pas d'aptitude artistique, [...] l'art qu'on lui prête vient des vaincus étrangers à sa race qu'il a fait travailler pour lui» (p. 393). C'est ce qui fait dire à juste titre à Jacques Marx, qui a beaucoup écrit à propos de ce récit de voyage, qu'avec Picard on touche à l'apothéose du regard dénigrant, inaugurant le « temps du mépris » dans l'histoire entre l'Occident et le Maghreb ${ }^{18}$.

\section{Stratégies discursives pour dénier la haine}

Dans ce texte où les images qui fomentent le mépris sont nombreuses, il est intéressant de remarquer une série de stratégies discursives qui cherchent à faire passer les théories raciales de l'auteur autrement que comme une obsession dictée par la haine. Il me semble qu'on peut les partager en deux ensembles différents.

D'une part, Picard insiste sur l'objectivité de ce qu'il reporte. Cette objectivité repose d'abord sur l'observation directe des réalités décrites: l'auteur atteste des «choses vues ", insiste sur sa sincérité et déclare vouloir se distinguer des récits où domine la «fausse turquerie » et l'« orientalisme de convention» $(p .160)^{19}$. Elle est ensuite liée au discours scientifique sur lequel Picard appuie une partie de ses réflexions; les allusions répétées à l'« étroitesse » de la race sémite, qui aurait l'un de ses fondements dans l'étroitesse du crâne de ses représentants, visent précisément à inscrire le discours picardien dans un cadre scientifique partagé et partageable, où les sentiments ne sont pas de mise ${ }^{20}$.

On remarque, d'autre part, une attitude constante que je nommerais "sur la défensive " et qui comporte l'attribution du rejet à l'autre, à l'accusé, et l'adoption d'une posture discursive où le sujet ne semble esquisser qu'une forme de défense face à une agression attestée. Autrement dit, au lieu d'exprimer haut et fort sa haine pour les sémites, Picard leur attribue des traits haïssables et, pour masquer encore plus sa propre agressivité, il tend à leur donner la parole, pour qu'ils soient les directs responsables de cette haine qu'ils suscitent. Le mécanisme qui sera systématique dans Jéricho, la pièce où Picard donne la parole, littéralement, à toute une famille de juifs, est déjà testé dans El Moghreb al Aksa, où l'auteur laisse souvent que les sémites se présentent eux-mêmes par des traits qui tendent, finalement, à les discréditer aux yeux du public européen (et le genre qu'il a choisi, le récit de voyage, lui donne une garantie d'authenticité que la pièce n'a pas). C'est ainsi que Picard reporte par exemple les répliques de l'entretien qu'il a eu avec Mohammad Amar, un chef du Riff, qui assume sa diversité raciale, oppose la « vie simple » des Marocains à la "vie compliquée » des Européens et appuie, de fait, les théories que l'auteur a jusque-là exposées :

Je lui demande : - Que pensez-vous de tout ce que vous avez vu là ? - C'est curieux. - N'en voudriez-vous pas au Maroc? - Non, c'est inutile. - Mais au contraire, cela nous sert. - Pour votre vie compliquée, pas pour notre vie simple. - Notre manière de vivre ne vous parait-elle pas meilleure ? - Non, on n'y a pas de repos. [...] - Ne voudriez-vous pas au moins notre bien-être, nos maisons, nos vêtements, notre nourriture? - Penser à cela causerait trop de soucis; nous en avons peu, ce qui donne le bonheur sur la terre, et diminue les occasions de pécher, ce qui promet le paradis. [...] - Votre civilisation vous semble donc supérieure à la nôtre ! - Oui, pour notre race; vos habitudes et vos besoins sont misérables parce qu'ils nous paraissent indignes d'un homme; il vous faut mille choses dont nous nous passons 
[...]. - Mais quelles grandes idées avez-vous ? - Notre religion, qui intervient dans toutes les actions de notre existence. - En définitive comment expliquez-vous que des millions d'Européens font pourtant ce que vous méprisez? - Mash Allah! Pourquoi le Datura porte-t-il des fruits vénéneux, tandis que l'herbe n'a que son herbe? (p. 225)

On peut très bien reconnaître la professionnalité de l'avocat qu'est Picard dans cette technique consistant à donner la parole à l'accusé, lui posant les bonnes questions pour qu'il réponde comme on voudrait qu'il le fasse. Et, de toute manière, l'auteur du récit de voyage aura toujours cet avantage sur ses personnages, qu'ils ne peuvent pas vraiment répliquer, et qu'il peut donc leur mettre dans la bouche tout et n'importe quoi.

En plus, comme je le disais, Picard attribue volontiers le rejet à l'autre. On ne compte pas les passages où, dans El Moghreb al Aksa, il présente des sémites intolérants vis-à-vis des aryens. Les musulmans détestent les chrétiens, «ces fils de chiens, ces Roumi, ces Nazrani » (p. 34) et, plus en général, les arabes haïssent les Européens (p. 236-237). Mais les juifs ne sont pas moins hostiles, au contraire :

Puis il y a les Juifs, surtout les Juifs, unis en franc-maçonnerie contre les Chrétiens (Oh! la joie de sentir les Chrétiens sous ses babouches!), formant cordon pour empêcher les intrus [...], s'entendant comme larrons en intelligence avec leurs coreligionnaires d'Europe [...]. Ils doivent coiffer la vile calotte noire, ils doivent marcher pieds nus quand ils sortent de leur quartier [...]. Mais ils sont les maîtres quand même [...]. Ce sont eux qui conquièrent le Maroc. La nation qui les aura avec elle y dominera. (p. 130-131)

Grâce à ces allusions à la rancœur des juifs à l'égard des chrétiens, à leur rapacité, au risque de leur invasion sournoise, le discours de la haine se camoufle en discours de défense. À vrai dire, Picard va même un peu plus loin, dans El Moghreb al Aksa. Il est vrai que son propos concerne le passé, mais le spectre du massacre ne se profile pas moins, lorsqu'il évoque l'erreur des Romains «de croire que, sans exterminer le Sémite, ils pourraient, eux Aryens, dominer le monde africain du Nord» (p.290). C'est la seule allusion dans ce sens, mais elle est là, et je la reporte.

Le refus de l'aryen de la part de cette terre qui le fascine chaque jour davantage est véritablement une obsession pour Picard, selon lequel même le paysage, les villes, et jusqu'au climat, exprimeraient leur hostilité vis-à-vis de l'ambassade en visite. C'est ainsi qu'il voit la ville de Meknès, « [s]uperbe en son étalement, respirant du silence, dressant ses minarets, ouvrant l'œil de ses embrasures, pour guetter notre exode morose et nous faire de hauts gestes de méprisant congé ", et sent les "écharpes » d'une averse les cingler «comme si tout le fanatisme de l'antique cité s'acharnait contre nous" (p. 340-341).

\section{Au carrefour des genres, la haine qui ne dit pas son nom}

30 La haine qui nourrit ce texte se traduit donc dans une série d'images prégnantes et se cache en même temps grâce à des stratégies discursives efficaces. Lorsque je dis " efficaces » je ne veux pas dire rigoureuses, au contraire : passés au crible de l'analyse, les raisonnements de Picard ne tiennent pas debout, ses arguments scientifiques ne s'appuient pas sur des bases suffisamment solides, l'auteur n'est pas toujours cohérent. Mais il peut se le permettre, du moment qu'il n'écrit pas un essai scientifique, qui l'exposerait à une série de critiques objectives, ou encore à une polémique, qui 
demanderait à entrer dans un débat ouvert à d'autres intervenants. El Moghreb al Aksa est un récit de voyage, un macro-genre qui tend volontiers à englober plusieurs types de discours. Au tournant du $\mathrm{XX}^{\mathrm{e}}$ siècle, lorsqu'il est question de l'Afrique et des colonies, le récit de voyage est d'ailleurs le genre auquel se greffent tous les autres genres, tous les autres discours. Des reportages de Henry Morton Stanley au Voyage au bout de la nuit de Céline, en passant par Au cœur des ténèbres de Joseph Conrad, le discours sur l'Afrique adopte la structure d'un récit de voyage (peu importe le degré de réalité de cet objet). Dans les dernières décennies du XIX ${ }^{e}$ siècle, la charpente formelle du genre est assez figée mais, à l'intérieur de cette maquette, le genre se prête à accueillir des contenus très variés.

Le récit de Picard se situe ainsi au carrefour de plusieurs genres : par exemple, on l'a vu, ses réflexions sembleraient convenir à un essai scientifique (ce n'est pas pour rien qu'une partie de celles-ci seront reprises dans des essais ultérieurs) quitte à dévier, d'autres fois, vers un journal beaucoup plus personnel. Ce discours qui se voudrait objectif, mais qui est soutenu par une subjectivité qui s'exprime de manière très émotive, pour ne pas dire vindicative, fait pencher le texte du côté du pamphlet ${ }^{21}$, et les tirades contre les sémites ou en défense des aryens ont souvent le ton d'une harangue juridique, qui reste cependant très familière et adopte les tons de la conversation. De plus, le discours de la haine tantôt s'affirme haut et fort et tantôt se dissout dans une atmosphère presque de rêve : n'oublions pas que l'auteur dit, en ouverture de son texte, avoir été enlevé par la fantaisie, et le conclut en s'exclamant : « Mon rêve est fini !» (p. 427).

L'efficacité discursive du récit me semble, en conclusion, intimement liée à ce mélange : le traité scientifique et la harangue juridique persuadent, le pamphlet émeut et scandalise, le récit de voyage rend tous ces discours acceptables, voire agréables à lire, grâce aussi au recours à l'art de la conversation et à un style artiste, d'esthète, qui a été défini

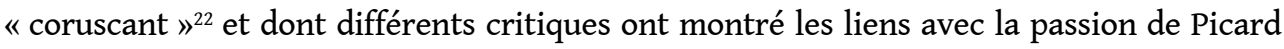
pour les arts figuratifs ${ }^{23}$. Au carrefour des genres et enveloppée dans un style artiste fin de siècle, la haine ne dit (presque) pas son nom et ne manque pas de pouvoir de séduction.

\section{NOTES}

1. P. Aron, C. Vanderpelen-Diagre, Edmond Picard (1836-1924). Un bourgeois socialiste belge à la fin du dix-neuvième siècle. Essai d'histoire culturelle, Bruxelles, Musées royaux des Beaux-Arts de Belgique, 2013.

2. Encore aujourd'hui, la page française de Wikipedia consacrée à l'auteur fait juste une allusion rapide à ses propos "jugés racistes, fascistes, antisémites" (c'est moi qui souligne), URL : < https://fr.wikipedia.org/wiki/Edmond_Picard>, consulté le 10/12/2016; la fiche sur Picard (brève, il est vrai) rédigée par Antoine Compagnon pour l'Encyclopedia Universalis en ligne ne mentionne, quant à elle, ni le racisme ni l'antisémitisme de l'auteur, URL : <http:// www.universalis.fr/encyclopedie/edmond-picard>, consulté le 10/12/2016.

3. M. Graindorge, Edmond Picard au Rwanda, Bruxelles, Le Cri, 1994 ; F. Ringelheim, Edmond Picard, jurisconsulte de race, Bruxelles, Larcier, 1999 ; P. Aron, C. Vanderpelen-Diagre, op. cit. 
4. E. Picard, El Moghreb al Aksa. Une mission belge au Maroc [Bruxelles, 1889], Bruxelles, Paul Lacomblez, 1893, URL : <https://archive.org/details/elmoghrebalaksa00picagoog>. La pagination de ce livre sera désormais citée directement dans le texte sans autre indication.

5. Plus précisément dans le numéro de septembre 1888, p. 245-267.

6. Voici les références bibliographiques complètes des textes cités: Synthèse de l'antisémitisme, Bruxelles/Paris, F. Larcier/A. Savine, 1892 ; L'Aryano-sémitisme, Bruxelles, Lacomblez, 1898 ; Jéricho: comédie-drame en trois actes et XXIV scènes, Bruxelles, Paul Lacomblez, 1902 ; Le Droit pur, cours d'encyclopédie du droit; les permanences juridiques abstraites [Bruxelles, 1899], Paris,

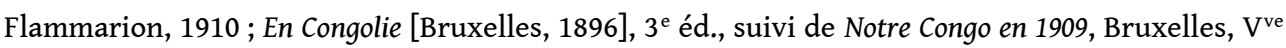
Ferdinand Larcier, 1909.

7. Cf. J. Marx, « Regards fin de siècle sur le Maghreb sombre », dans P. Halen, J. Riesz (dir.), Images de l'Afrique et du Congo-Zaïre dans les Lettres françaises de Belgique et alentour, Bruxelles, Textyles Éditions / Kinshasa, éditions du Trottoir, 1993, p. 189-204 ; Id., "Visions d'Afrique du Nord dans les lettres françaises de Belgique », Textyles, n. 12, 1995 (Voyages, ailleurs), p. 47-66 ; Id., « L'Afrique du Nord dans la littérature belge ", site de la Société internationale d'étude des littératures de l'ère coloniale (SIELEC), URL: <http://www.sielec.net/pages_site/DESTINATIONS/MAGHREB/ Marx_afr_litt_belge/Marx_afr_litt_belge_1.htm>, consulté le 16/11/2016.

8. Cf. J. Marx, « Visions d'Afrique du Nord dans les lettres françaises de Belgique », cit., p. 53.

9. Ibid., p. 52.

10. Id., "Regards fin de siècle sur le Maghreb sombre », cit., p. 191. On remarquera qu'il n'y a presque pas de trace d'antisémitisme dans le récit $A u$ Maroc de Loti, alors qu'il y en aura dans Jérusalem, un autre récit de voyage qu'il publie cinq ans plus tard, en 1895.

11. Vers les trois quarts du livre, on peut encore lire : «Mon livre sera pour quelques amis chers... et pour moi, pour refaire mon voyage au retour » (p. 315).

12. Cf. G. Jucquois, P. Sauvage, L'Invention de l'antisémitisme racial. L'implication des catholiques français et belges (1850-2000), Bruxelles, Academia Bruylant, « Sciences et Enjeux », 2001, p. 124-125. 13. Cf. aussi J.-F. Six, 1886. Naissance du XX $X^{e}$ siècle en France, Paris, Seuil, 1986, p. 161.

14. Cf. R. Amossy, E. Rosen, Les discours du cliché, Paris, SEDES, 1982 ; R. Amossy, Les Idées reçues: sémiologie du stéréotype, Paris, Nathan, 1991 ; A. Goulet (dir), Le Stéréotype : crise et transformations, Colloque de Cerisy-la-Salle (7-10 octobre 1993), Caen, Presses Universitaires de Caen, 1994.

15. Paul Aron et Cécile Vanderpelen-Diagre ont raison, par exemple, lorsqu'ils invitent à donner au racisme de Picard une dimension où ne pèse pas l'évolution que le phénomène a connue au XX e siècle (P. Aron, C. Vanderpelen-Diagre, Edmond Picard (1836-1924), op. cit.).

16. E. Saïd, L'Orientalisme. L'Orient créé par l'Occident [London, 1978], Paris, Seuil, 1980.

17. Cf. entre autres M. C. Gnocchi, «Le Blanc, le Noir et... l'Autre. Stratégies discursives coloniales (1875-1914)», dans J.-F. Durand, J.-M. Seillan (dir.), L'Aventure coloniale, Paris, Kailash, «Les Cahiers de la SIELEC », 2011, p. 418-430.

18. Cf. J. Marx, « Regards fin de siècle sur le Maghreb sombre », cit., p. 192. Marx doit l'expression "temps du mépris " à Abdeljalil Lahjomri, qui s'en sert dans son essai L'image du Maroc dans la littérature française de Loti à Montherlant, Alger, SNED, 1973, réédité sous le titre Le Maroc des Heures françaises, Casablanca, Marsam/Stouky, 1999.

19. L'attitude de Picard est celle du « reporter » qui s'affirme à la fin du XIX ${ }^{\mathrm{e}}$ siècle dans la presse française, et donc du témoin qui privilégie le «journalisme du voir» par rapport au «journalisme du dire», cf. G. Muhlmann, Une histoire politique du journalisme (XIX ${ }^{e}$-XX $X^{e}$ siècle), Paris, Le Monde/Presses Universitaires de France, «Partage du savoir », 2004, réédition Seuil, «Points », 2007. Lorsqu'il fustige les récits « orientalistes» trop fantaisistes, il vise surtout les auteurs d'autres récits d'ambassade au Maroc, premier entre tous Gabriel Charmes, qui avait publié Une ambassade au Maroc chez Calmann-Lévy en 1887 et qu'il cite plusieurs fois, toujours pour le démentir, dans les pages d'El Moghreb al Aksa. 
20. Bien conscient de l'ambiguïté de son discours, dans L'Aryano-sémitisme Picard invitera ses lecteurs - par une sorte d'excusatio non petita - à voir dans son antisémitisme autre chose qu'« un système d'antagonisme religieux ou une prédication de haine" (E. Picard, L'Aryano-sémitisme, op. cit., p. 15).

21. M. Angenot, La Parole pamphlétaire. Typologie des discours modernes, Paris, Payot, 1982.

22. P. Delsemme, "Le style coruscant ", dans La littérature fin de siècle. Une littérature décadente?, numéro spécial de la Revue luxembourgeoise de littérature générale et comparée, 1990, p. 149-159.

23. Jacques Marx n'a pas tort lorsqu'il remarque que la dimension esthétique camoufle, dans $E l$ Moghreb al Aksa, le projet idéologique de l'auteur (J. Marx, « Regards fin de siècle sur le Maghreb sombre ", cit., p. 193-194). Ce mélange de genres opère un brouillage dans le cadre pragmatique, au niveau des actes du langage impliqués, exactement comme cela se passe chez Céline, selon Philippe Roussin. Roussin explique en effet que les pamphlets de Céline « se font passer pour des actes de langage engageant l'émotion et l'opinion de l'émetteur ; ils sont en réalité le moyen de provoquer des changements dans le monde et d'y associer le lecteur » ( $\mathrm{Ph}$. Roussin, Misère de la littérature, terreur de l'histoire. Céline et la littérature contemporaine, Paris, Gallimard, " NRF essais ", 2005, p. 458)

\section{RÉSUMÉS}

Avocat, sénateur socialiste, essayiste, journaliste, amateur d'art, animateur de la vie culturelle bruxelloise, Edmond Picard (1836-1924) a également été un théoricien débridé de l'antisémitisme et du racisme. Le premier texte dans lequel l'auteur développe ces théories est El Moghreb al Aksa. Une mission belge au Maroc, un récit de voyage publié pour la première fois en 1889, qui se révèlera une matrice importante pour bien d'autres œuvres de sa plume, voire un modèle pour d'autres auteurs. Cet article s'interroge sur les formes discursives que prend la haine dans cette œuvre. L'auteure y repère une série de représentations de l'« autre " (sémite), plus ou moins imagées, accompagnées par des stratégies discursives finalisées à la dénégation de cette même haine, et suggère que l'efficacité discursive du récit doit beaucoup au fait qu'il se situe au carrefour de plusieurs genres.

\section{INDEX}

Mots-clés : antisémitisme, racisme, récit de voyage, Maroc, littérature belge, haine, rhétorique 